\title{
Classification of problematic Internet usage types by motives and contexts with elementary and secondary school-aged counseling clients
}

\author{
BUGEUN KIM ${ }^{1}$, SEUL LEE ${ }^{2}$, YOUNG YIM DOH ${ }^{3}$ and GAHGENE GWEON ${ }^{1 *}$ \\ ${ }^{1}$ Department of Transdisciplinary Studies, Seoul National University, Seoul, Republic of Korea \\ ${ }^{2}$ Cognitive Computing Lab, Advanced Institute of Convergence Technology, Suwon, Republic of Korea \\ ${ }^{3}$ Graduate School of Culture Technology, Korea Advanced Institute of Science and Technology (KAIST), Daejeon, Republic of Korea
}

(Received: November 28, 2017; revised manuscript received: June 28, 2018; accepted: August 12, 2018)

\begin{abstract}
Background and aims: Past research on the classification of problematic Internet use (PIU) has focused on symptombased severity and usage motive in order to understand its mechanism. Recently, usage context, such as family or social relationships, has been identified as a key influencing factor of PIU. Therefore, we extended the classification of PIU to include usage context in addition to symptom-based severity and usage motive. Methods: To classify PIU types, we conducted two studies. First, we performed a clustering analysis, where 265 counseling cases were clustered into usage types in terms of motive and context. After characterizing each usage type, we examined their hierarchical relationships by considering symptoms. Second, we performed a focus group interview with six counselors to increase the transferability of usage types. This transferability was established by matching counselors' quotations to the usage types. When usage types showed consistency between quotations and the clustering analysis, we identified the progression patterns between hierarchical relationships. Results: The clustering analysis of motive and context yielded six usage types with three hierarchical relationships. The focus group interview results verified the transferability of these six types and identified two progression patterns between the hierarchical relationships. Discussion and conclusion: Although usage motive and context were given equal weight before the clustering analysis, the resulting types revealed that usage context played a greater role in the classification process.
\end{abstract}

Keywords: problematic Internet use, usage types, usage context, usage motive, symptom, elementary and secondary school-aged counseling clients

\section{INTRODUCTION}

There have been steady attempts to understand problematic Internet use (PIU) by classifying the users' behavior into usage types (Caplan, 2002; Kim \& Kim, 2010; Laconi, Tricard, \& Chabrol, 2015; Wu, Lai, Yu, Lau, \& Lei, 2017; Young, 1998; Young, Pistner, O’Mara, \& Buchanan, 1999). Classifying the usage types provides an analytical framework to study the relationships between the underlying factors that influence PIU and trace its progression patterns. Previous studies have provided a tool for identifying the usage types based on two classification criteria: symptombased severity and usage motive. However, usage context, which is an individual's perception of their circumstances, has not been deeply considered in usage classification research so far. Given that the usage context has been shown to influence PIU, in this study, we sought to explore the importance of considering usage context in usage type classification. To study the context, we adopted the cyclic value-context reinforcement model of Internet use behavior (CVCRM; Doh, Lee, Kim, \& Gweon, 2018), which integrates existing theoretical approaches (Davis, 2001; Song, LaRose, Eastin, \& Lin, 2004) and highlights the three interacting aspects of psychosocial value, environmental context, and Internet utility in describing the reinforcement cycle of PIU, as shown in Figure 1.

Existing work on the classification of PIU has mainly focused on using symptom-based severity or usage motive as classification criteria. Regarding symptom-based severity, researchers were able to distinguish between problematic and non-problematic usage based on the presence of negative symptoms (Young, 1998). Another symptom-based classification focused on the non-problematic, highengagement activities of computing (Charlton \& Danforth, 2004). Since symptoms vary across cultures, scales have been developed or translated to address the contexts of different cultures, such as South Korea, China, Italy, and Persia (Huang, Wang, Qian, Zhong, \& Tao, 2007; Monacis, de Palo, Griffiths, \& Sinatra, 2016; National Information Society Agency, 2011; Wu, Lin, et al., 2017). In addition, scales have been developed to measure symptoms occurring in the context of different media, such as online games, smart media, and social networking sites

* Corresponding author: Gahgene Gweon; Department of Transdisciplinary Studies, Seoul National University, 1 Gwanak-ro, Gwanak-gu, Seoul 08826, Republic of Korea; Phone: +8231 888 9172; Fax: +82 31888 9159; E-mail: ggweon@snu.ac.kr

This is an open-access article distributed under the terms of the Creative Commons Attribution-NonCommercial 4.0 International License, which permits unrestricted use, distribution, and reproduction in any medium for non-commercial purposes, provided the original author and source are credited, a link to the CC License is provided, and changes - if any - are indicated. 


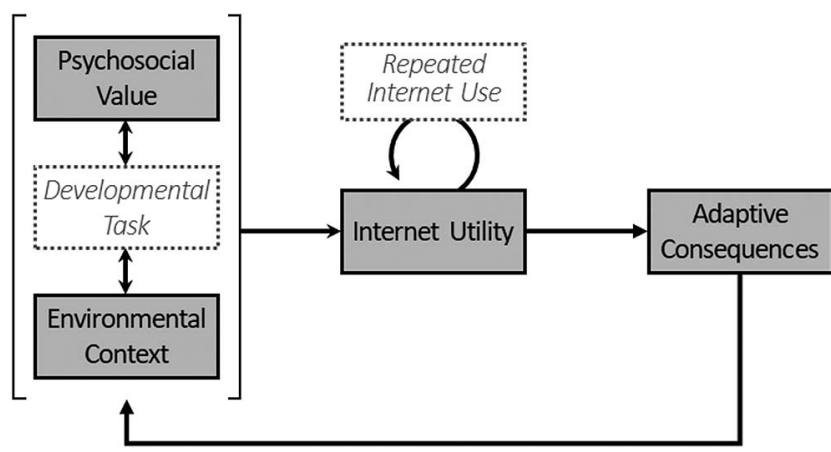

Figure 1. The cyclic value-context reinforcement model of Internet use behavior (Doh et al., 2018). The graphical model is slightly simplified to emphasize the aspects that we are using in the present paper; "environmental context" (which corresponds to our usage context), "psychosocial value" and "Internet utility" (motive), and "adaptive consequence" (symptom)

(Kim \& Kim, 2010; Marino, Vieno, Altoe, \& Spada, 2017; Smetaniuk, 2014).

As for usage motive, researchers have classified motives for playing online games (Yee, 2006) or social casino games (Gainsbury, King, Russell, \& Delfabbro, 2016). Researchers have also developed theories to explain usage motives in terms of maladaptive cognitions (Davis, 2001) or gratification from the usage (Song et al., 2004). These motives and gratification theories have inspired the development of several scales for classifying PIU based on motives (Kneer \& Glock, 2013; Wu, Lai, et al., 2017) or underlying cognitions (Caplan, 2002).

Although the research on classifying PIU according to symptom-based severity and usage motive has provided insight into the underlying factors of PIU, the importance of usage context - that is, the circumstances that affect or interact with usage behavior - has been mostly overlooked. Considering usage context will provide a PIU model that offers a more complete picture of users' Internet use mechanisms, which, in turn, can influence the overall understanding of PIU and the design of PIU interventions. For example, let us assume that two users $-A$ and $B$ - have the same motive (entertainment and social belongingness) and symptom-based severity (high risk). However, the context in which $A$ and $B$ use the Internet differs. $A$ primarily uses the Internet with offline friends, utilizing it as a tool to help maintain her friendships with them. $B$, however, mostly uses the Internet at home, because she wants to meet others online and seek the social belongingness that she cannot obtain in daily life because of domestic disturbances. Owing to these contextual differences, the interventions for relieving PIU should differ $-A$ needs counseling about her friendships, whereas $B$ requires counseling about her domestic disturbances. As illustrated by these cases, understanding usage context by differentiating between user subtypes can provide further insight into PIU.

Although usage context has not been studied in relation to PIU classification, existing literature has nevertheless verified its importance as an influencing factor of PIU. Context has been used to explain motives for problematic usage (Snodgrass, Dengah, Lacy, \& Fagan, 2013) and to investigate the progression of PIU (Griffiths, 2010).
Moreover, researchers have suggested considering usage context when trying to understand problematic usage (Karlsen, 2013) or examining the meaning of the Internet for users (Kuss, 2013). Hence, in line with previous research on usage context, this study utilizes three types of contexts (individual, familial, and societal), which interact with usage behavior and influence daily life. In this study, the "individual context" refers to a user's frame of mind, which is an internal psychological state that is affected by both the familial and societal contexts. Examples of individual contextual factors from previous research include academic stress and life satisfaction (Jun \& Choi, 2015; Laconi et al., 2015; Pawlikowski, Nader, Burger, Stieger, \& Brand, 2014). Note that psychological traits (e.g., individual characteristics, psychiatric diseases, and self-concepts) are not included in the individual context, because they do not directly reveal how the user is affected by his or her circumstances. The "familial context" and "societal context" refer to users' evaluations of their family and society, respectively. These two contexts fit into the "microsystem" of ecological systems theory (Bronfenbrenner, 1979). Examples of familial factors include parent-adolescent conflict, family communication, parenting attitudes, and family violence exposure (Yen, Yen, Chen, Chen, \& Ko, 2007; Park, Kim, \& Cho, 2008; Liu, Fang, Deng, \& Zhang, 2012). Examples of societal factors include loneliness, deviant peer affiliations, perceived social support, and socioeconomic variables (Gunuc \& Dogan, 2013; Kim, LaRose, \& Peng, 2009; Ko et al., 2008; Lai \& Kwan, 2017; Li et al., 2013).

By considering motive and context together, we attempt to answer the following research question: What are the PIU usage types and the characteristics of each type? In addressing this research question, we used a mixed-methods approach to increase transferability. In "Methods" section, we introduce two studies: clustering analysis and focus group interview. The "Results" section reports on the usage types identified from the clustering analysis and the quotations corresponding to each type from the focus group interview. Finally, we discuss the meaning and underlying relationships between the types under "Discussion" section.

\section{METHODS}

To address our goal of classifying usage types, we used a systemic approach, which examines prior causes or contexts that trigger symptoms, rather than a symptomatic approach that solely focuses on alleviating the symptoms. In other words, to identify prior contexts through classification, we focused solely on usage motive and context, not on symptoms. However, we considered symptoms in examining how different PIU types affect each other over time through exploration of their hierarchical relationships and progression patterns. We conducted two studies as shown in Figure 2: a clustering analysis using 265 counseling cases to identify usage types and examine hierarchical relationships and a focus group interview with six counseling experts to increase the transferability of the 
identified types and identify progression patterns in actual counseling settings.

\section{Measures}

To address our research goal of usage type classification, we extracted the following four measures: symptom-based severity, usage motive, context, and symptoms. First, to measure the symptom-based severity of the 265 counseling cases, we used the K-scale, which was developed by the Korean government (National Information Society Agency, 2011). This 15-item scale is actively used in South Korea by Internet addiction counselors and was validated by Lee et al. (2013). The K-scale classifies counseling cases into three severity levels: normal, potential, and high risks.

Second, to measure usage motive, context, and symptoms, the CVCRM (Doh et al., 2018) was used. The CVCRM is a theoretical framework explaining the developmental mechanism of Internet usage as shown in Figure 1. The CVCRM proposes four aspects: "environmental context," "psychosocial value," "Internet utility," and "adaptive consequences." We grouped psychosocial value and Internet utility in the "usage motive" category, since the differences between these categories were subtle. Therefore, the factors under psychosocial value and Internet utility were not differentiated in either the clustering analysis or focus group data. The resulting three categories were renamed to highlight the "usage" aspect of PIU. (a) The usage motive category (consisting of the psychosocial value and Internet utility categories of the CVCRM) refers to the psychological factors motivating actual Internet usage. Psychosocial value refers to a user's intrinsic needs that are satisfied by Internet usage. Internet utility refers to the utility of the Internet in satisfying the psychosocial value of a user. (b) We renamed the environmental context category of the CVCRM, defined as the circumstances affecting a user's perception or behavior, as usage context. Thus, the usage context category includes factors that interact with usage behavior and influence the daily lives of a user. (c) The adaptive consequence category of the CVCRM, which refers to the negative results of Internet use, was renamed as "symptoms." Thus, the symptoms category describes the negative symptoms caused by PIU.

\section{Clustering analysis}

To identify the usage types of PIU, we followed the four steps of clustering analysis as shown in Figure 2A. First, we prepared four measurements: usage motive, context, symptoms, and symptom-based severity, using the CVCRM and K-scale. Second, the cases were then clustered by hierarchical clustering on principal components (HCPC; Husson, Josse, \& Pages, 2010) with usage motive and context factors. Third, we identified the characteristics of usage types using the $\chi^{2}$ test for motive and context factors. Finally, we examined hierarchical relationships of the types by comparing their symptoms using a $t$-test.

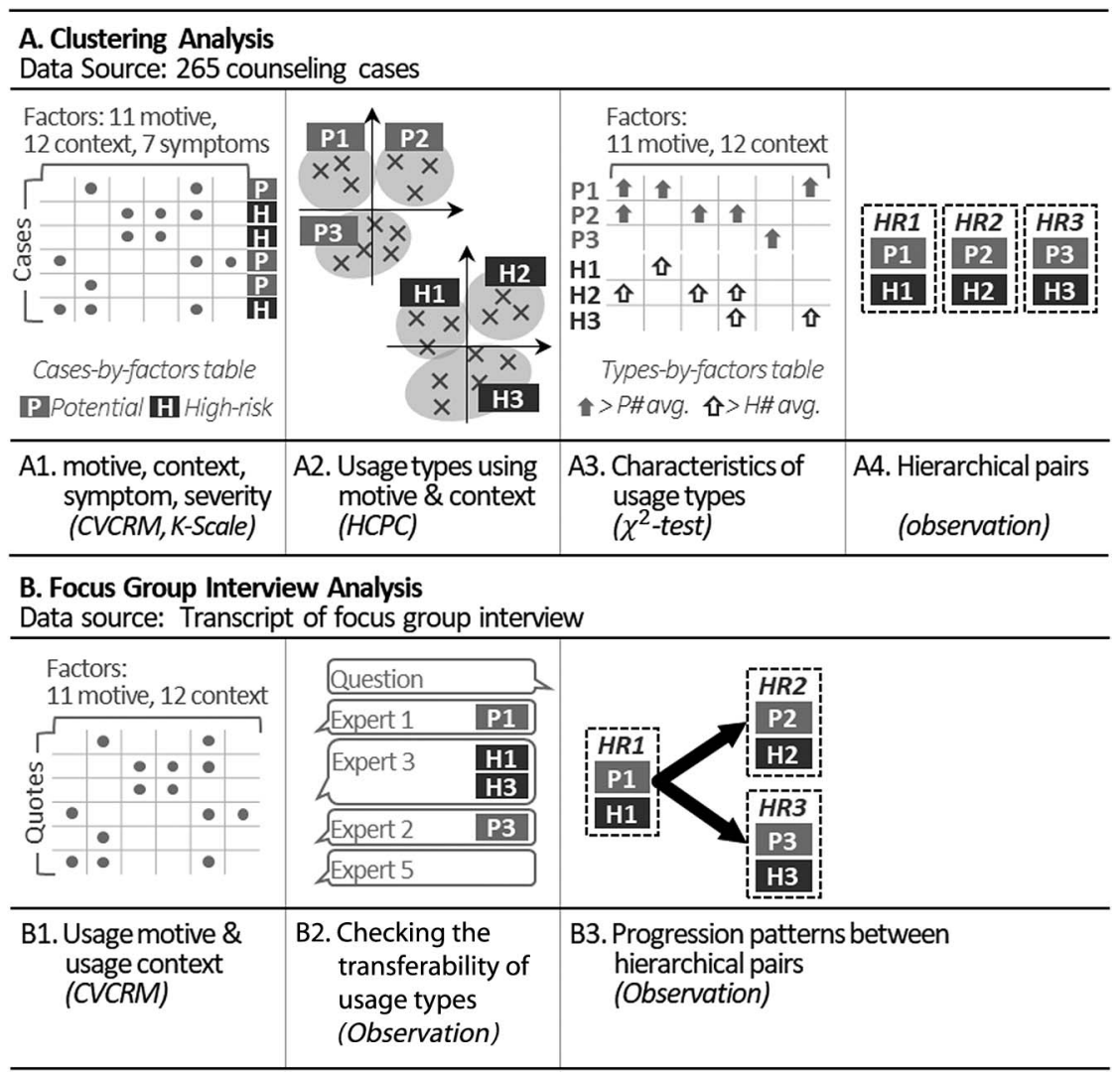

Figure 2. Two studies conducted in this paper. (A) shows three steps of clustering analysis that identifies usage types and hierarchical relationships, with their expected outputs. (B) shows three steps of focus group interview analysis that increases transferability of identified usage types 


\section{Participants and data source}

We examined the Internet usage patterns of 265 individual counseling cases of elementary and secondary school-aged counseling clients. The counseling sessions were conducted by experts at the Internet Addiction Prevention Center (IAPC) of South Korea. Although the participants were predominantly male $(81.9 \%, n=217)$, they were evenly distributed in terms of age (8-19 years, mean $=13.8, S D=$ 2.6). All counseling sessions were held between January 2012 and May 2014. For each client's case, a counselor performed and documented an initial session inquiring about the usage motive, context, symptoms, and symptom-based severity using 42 predefined questions. The counselor conducted up to five follow-up counseling sessions when requested by the client, and each session was documented by answering two predefined questions summarizing observations and insights from the counseling session. In total, the counselors produced 2,036 counseling session documents for the 265 counseling cases.

\section{Procedure}

Prior to classifying usage types based on motive and context, we used the K-scale to divide the counseling cases according to their symptom-based severity. The division resulted in 113 potential-risk cases $(42.6 \%)$ and 152 highrisk cases $(57.4 \%)$. Within each severity level, we then used the 23 factors of motive and context to identify usage types using HCPC (Husson et al., 2010). In detail, the classification procedure consisted of the following four steps.

Step 1. We quantified usage motive, context, and symptoms by labeling the counseling cases using the 37 factors of the CVCRM. Six graduate student researchers coded the 265 counseling cases under the supervision of two professors. The research team adjusted the coding manual nine times to establish agreement between coders: the average agreement AC1 score (Gwet, 2008) across 37 factors was $0.879(\min =0.487$ and $\max =1.000)$. Of the original 37 factors proposed by the CVCRM, we removed seven factors that appeared in less than $5 \%$ of the data. The remaining 30 factors that we used for clustering the usage types are listed in Table 1. This coding process resulted in a dichotomous cases-by-factors table, as shown in Figure 2 step A1.

Step 2. We used the HCPC method to cluster the usage types. HCPC is a statistical analysis method for identifying clusters of similar instances when each instance is measured in categorical variables. To prepare the real-valued vector input for HCPC, we converted each row, which corresponds to one client's case, of the cases-by-factors table into a real-valued vector using multiple correspondence analysis (MCA; Greenacre \& Blasius, 2006). For example, in the cases-by-factors table, a potential-risk case (no. 1763) is represented as 1-0-0-1-0-0-0-0-1-0-0-0-1-1-1-0-1-1-0-0-01-1 (the corresponding 23 motive/context factors are shown in Table 1). Using MCA, similar factors are grouped together, resulting in a 19-dimensional vector for the potentialrisk cases. Rather than being a binary number, each dimension has a real value. Thus, the case no. 1763 becomes a 19-dimensional vector of $(0.3378,0.8898,0.2087, \ldots$, $-0.0904)$. Each real value represents the normalized weight
Table 1. List of 16 usage motives, 13 usage contexts, and 8 symptom factors adopted from cyclic value-context reinforcement model (Doh et al., 2018)

\begin{tabular}{|c|c|c|}
\hline \multirow[t]{2}{*}{$\begin{array}{l}\text { Usage } \\
\text { motive }\end{array}$} & Individual & $\begin{array}{l}\text { To relieve stressful events } \\
\text { To experience a sense of } \\
\text { accomplishment } \\
\text { To spend one's surplus time } \\
\text { To enjoy the contents themselves } \\
\text { To surf the Internet } \\
\text { To express passive resistance }{ }^{\mathrm{a}} \\
\text { To escape from reality } \\
\text { To satisfy one's sexual curiosity } \\
\text { To express oneself } \\
\text { To engage in goal oriented learning } \\
\text { To earn money }\end{array}$ \\
\hline & Societal & $\begin{array}{l}\text { To participate in common activities in } \\
\text { the peer group } \\
\text { To be active in an online community } \\
\text { To be recognized as the best }{ }^{\mathrm{a}} \\
\text { To develop a sense of closeness } \\
\text { To experience controllability }\end{array}$ \\
\hline \multirow[t]{3}{*}{$\begin{array}{l}\text { Usage } \\
\text { context }\end{array}$} & Individual & $\begin{array}{l}\text { Academic stress } \\
\text { Situations of insufficient satisfaction of } \\
\text { psychosocial needs } \\
\text { Lack of a lifelong goal } \\
\text { Physical illness that limits daily life }\end{array}$ \\
\hline & Familial & $\begin{array}{l}\text { Inadequate parenting } \\
\text { Family communication problems } \\
\text { Instability of the caregiver } \\
\text { Internet overuse by other family } \\
\text { members }\end{array}$ \\
\hline & Societal & $\begin{array}{l}\text { Socially imposed stigma } \\
\text { Social/school maladjustment } \\
\text { Economic constraints } \\
\text { Coexisting delinquent behavior } \\
\text { Unstructured daily activities }\end{array}$ \\
\hline \multirow[t]{2}{*}{ Symptom } & Individual & $\begin{array}{l}\text { Interruption of academic performance } \\
\text { Physical symptoms } \\
\text { Difficulty in controlling Internet use } \\
\text { Awareness of problematic usage } \\
\text { Lack of awareness of problematic } \\
\text { usage } \\
\text { Spending beyond one's ability to pay }\end{array}$ \\
\hline & Societal & $\begin{array}{l}\text { Conflicts } \\
\text { Weakening of/disconnection from } \\
\text { social relationships }\end{array}$ \\
\hline
\end{tabular}

Note. Italicized factors were excluded from this paper, since they appeared in less than $5 \%$ of the counseling cases.

${ }^{\mathrm{a}}$ Factors only appeared in high-risk cases.

of a particular dimension (e.g., the value of the first dimension in case no. 1763 is 0.3378 ) across all cases. Finally, HCPC clusters the real-valued vectors into usage types.

Step 3. We characterized the identified types. After identifying the types with HCPC, we conducted further analysis to characterize each type by identifying which of the 23 motive/context factors distinguished each type from the others. The characterization process was conducted 
within each severity level. For each identified type, a $\chi^{2}$ test was conducted on each of the 23 factors to verify whether its occurrence in a given type was greater than the overall occurrence in that severity level. For example, assuming that type $\mathrm{H} 1$ belongs to the high-risk level, a $\chi^{2}$ test was then performed on each of the 23 factors for this type - for instance, we compared the occurrence of "academic stress" in type H1 (e.g., 0.50) to its overall occurrence in the highrisk type (e.g., 0.257). This analysis resulted in a typesby-factors table, as shown in step A2 of Figure 2.

Step 4. The hierarchical relationships between the usage types were identified across different severity levels through observation and then verified using $t$-tests. For each potentialrisk type, the research team examined which high-risk type was most similar. To determine the similarity between types, we qualitatively examined the types-by-factors table, which was the result of $\chi^{2}$ tests. We labeled the matched potential-risk and high-risk types as a hierarchical relationship. Then, for each hierarchical relationship, we conducted a $t$-test to verify that the high-risk type had more symptoms than the corresponding potential-risk type. For example, if types $\mathrm{P} 1$ and $\mathrm{H} 1$ formed a hierarchical relationship, we compared the average number of symptoms in P1 (e.g., 2.82) and $\mathrm{H} 1$ (e.g., 3.33) using a $t$-test.

\section{Focus group interview}

We conducted a focus group interview with six counselors regarding their experience of PIU counseling sessions to increase transferability of the usage types from the clustering analysis. All six counselors had worked at the IAPC, which helps clients in managing PIU. Their counseling experience was between 4 and 8 years (average $=5.6$ years). The details of the focus group data analysis involved the following three steps, as shown in Figure 2B.

Step 1. The focus group interview lasted an hour and consisted of two main discussion points about counselors' impressions of PIU clients' characters. The counselors discussed the potential factors that are affecting PIU and were asked to identify the usage types of PIU. Since the goal of this focus group interview was to increase the transferability of the identified types based on counselors' experience, the types identified from our clustering analysis were only presented to counselors after they had presented their own usage types. All the counselors were then asked to evaluate the transferability of each of the six clustering analysis usage types based on their experience.

Step 2. To match counselors' quotes to the characteristics of identified types, we first transcribed the focus group session. The transcriptions were then coded using the same coding manual of the 30 CVCRM factors as described in step B2 of Figure 2. Two graduate students, who had participated in the coding procedure of the clustering analysis, coded the transcription data (AC1 score; mean $=0.98$, $\min =0.90$, and $\max =1.00)$. This analysis resulted in eight usage types: six were identical to the clustering analysis results and two newly identified usage types.

Step 3. For the six types that were commonly found in both the clustering analysis and step 2 above, two coders further examined the transcript to identify progression patterns, namely the relationships between hierarchical relationships. For each occurrence of a usage-type instance in step 2 , if two or more types were mentioned within the same context, the coders checked for a progressional relationship between the types. Whenever such a relationship existed, we labeled the relationship using the types involved (e.g., the H1-H2 or H1-H3 labels are provided in the coding instances shown in Table 4.).

\section{Ethics}

The study procedures were carried out in accordance with the Declaration of Helsinki. The institutional review board of the Seoul National University approved the study. All subjects were informed about the study and all provided informed consent when the Internet Addiction Prevention Center collected information.

\section{RESULTS}

The results are as follows: (a) six usage types and three hierarchical relationships were derived from the clustering analysis, and (b) the transferability of six usage types and two progression patterns were confirmed by the focus group interview. Figure 3 shows results of the two analyses.

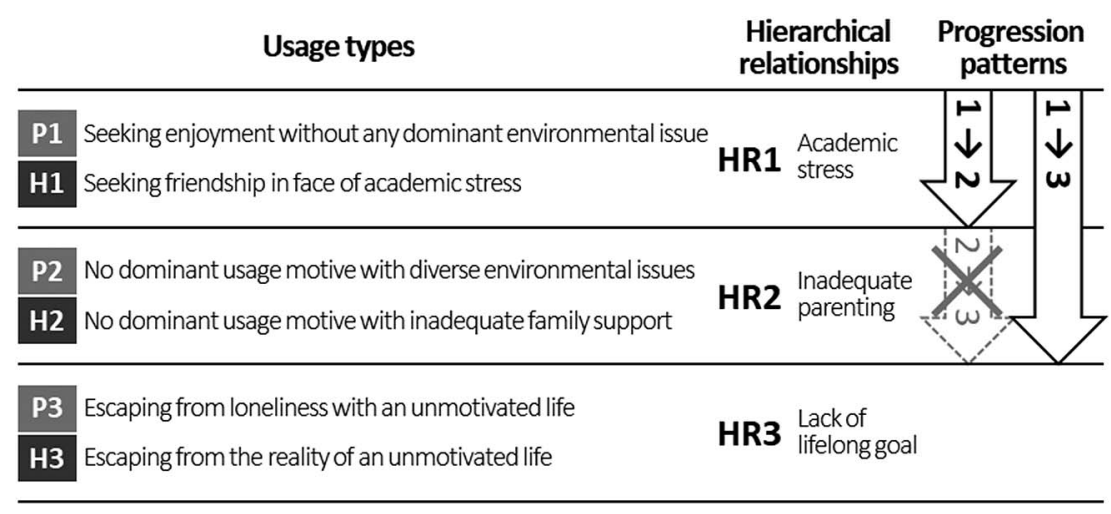

Figure 3. Hierarchical relationships and progression patterns between usage types 
Classification of problematic Internet usage

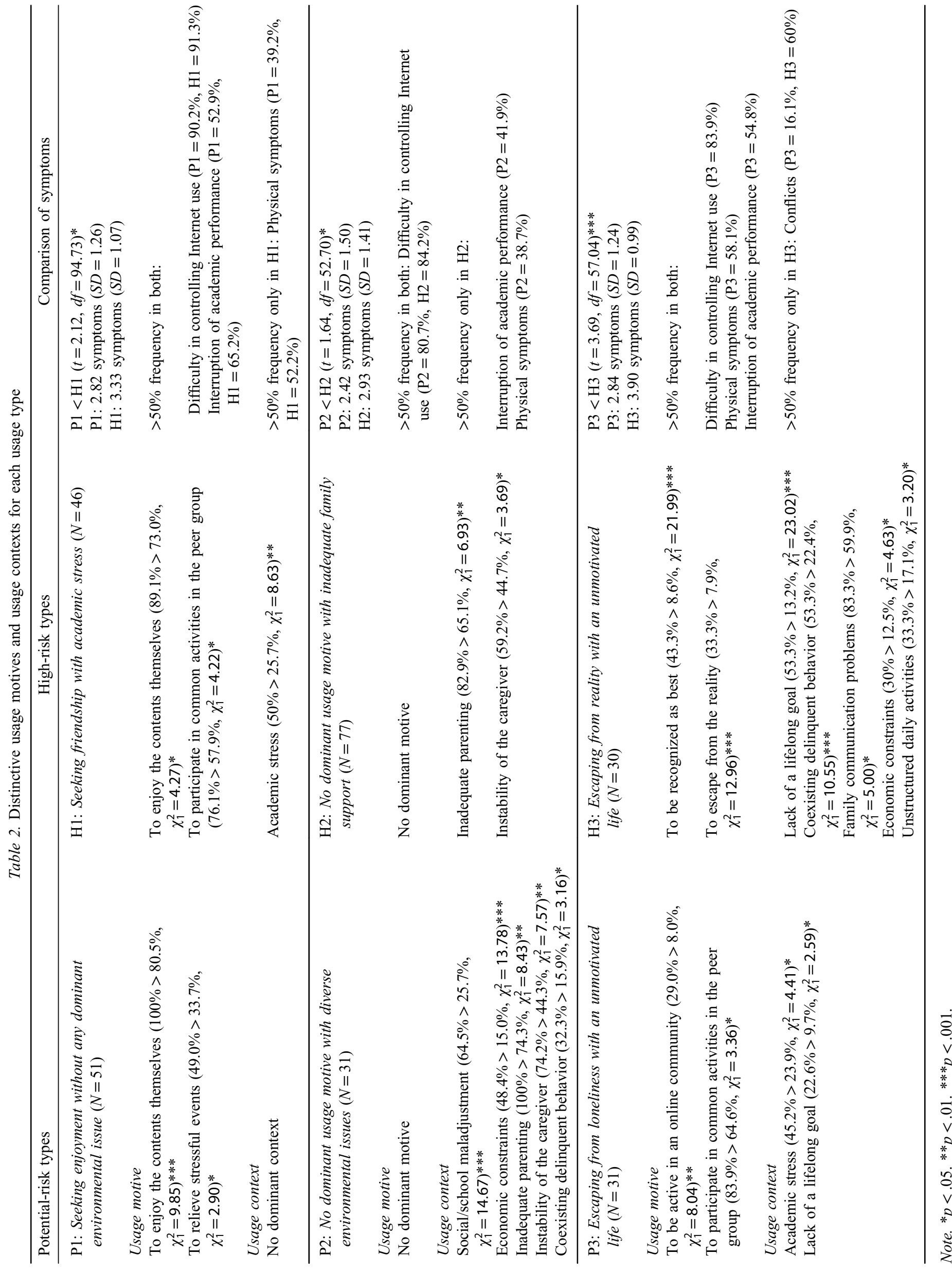


Identifying usage types from the quantified counseling cases

To identify the usage types of PIU using motive and context, we clustered the quantified counseling cases using HCPC. This analysis resulted in three potential-risk types and three highrisk types. We labeled these six types according to their dominant motive and context. The three potential-risk types are (P1) - seeking enjoyment without any dominant environmental issue, $(\mathrm{P} 2)$ - no dominant usage motive with diverse environmental issues, and (P3) - escaping from loneliness in an unmotivated life. Similarly, the three high-risk types are (H1) - seeking friendship in the face of academic stress, (H2) no dominant usage motive with inadequate family support, and (H3) - escaping from the reality of an unmotivated life. For each of the six types, distinctive motives and contexts are displayed in Table 2. Note that the types-by-factors table shown in step A3 of Figure 2 shows all 138 possible combinations of the 23 motive/context factors for the six types. Table 2 only lists the statistically significant motive and context factors identified from the series of $138 \chi^{2}$ tests.

We found that all six usage types fit into one of three hierarchical relationships: (HR1), the P1-H1 pattern, where academic stress leads to PIU; (HR2), the P2-H2 pattern, where family problems lead to Internet usage; and (HR3), the P3-H3 pattern, where the lack of a lifelong goal leads to Internet usage. In addition to motive and context, the third column in Table 2 lists the symptoms along with the $t$-test results, which show that the number of symptoms is higher in usage types at the high-risk severity level than at the potential-risk level.

\section{Verifying six usage types from the focus group interview data}

To examine the transferability of the six identified usage types from the counselors' viewpoints, we aligned the quotes of the focus group interview data with the usage types. All six counselors agreed that the six identified types were in line with their observations during counseling. In addition, we verified that all six types were found in the focus group interview transcriptions through line-by-line coding. During this coding process, we also obtained two additional usage types. Table 3 shows the quotes aligned with each of the six types in terms of motive and context. In addition, we identified two progression patterns from the focus group interview coding process, as shown in Table 4: (1) from HR1 to HR2 and (2) from HR1 to HR3.

Table 3. Sampled quotes from the focus group interview that are related to the six usage types and two additional types

P1: Seeking enjoyment without any dominant environmental issue

Despite the fact that they are doing homework and maintaining their grades, parents are not satisfied with their children's current status [since there is room for much improvement in their grades]. Parents don't want their children to spend time on the Internet [games] and want them to focus more on their studies.

P2: No dominant usage motive with diverse environmental issues

If clients have an insecure attachment [with parents] or [ . . ] had some traumatic experience in a peer relationship, it's more likely that they will engage more and more with [online] games [to spend their time in solitude].

\section{P3: Escaping from loneliness in an unmotivated life}

Some clients feel frustrated and stressed about [their academic performance], which they wanted to achieve but couldn't achieve. They do not have the ability to fight against such stress and [...] so they begin to be dependent on online games [to forget the feeling of frustration temporally with their friends].

H1: Seeking friendship in the face of academic stress

[While talking about the parents who have excessive academic expectations of their children] As their children grow up, since they frequently play games with friends, conflicts about their academic performance arise between the parents and their children. [...] As the parents are deeply concerned about their children's academic performance, conflicts become intense.

H2: No dominant usage motive with inadequate family support

Some clients do not go out because they have difficulty in interpersonal relationships [with parents and friends] or are depressed. However, as there are not many things that they can do at home, they play internet games or use their smartphones. [...] After a year or two of such behavior, it becomes difficult for them to go out or adjust in school.

H3: Escaping from the reality of an unmotivated life

Children begin to face reality as they grow older. [...] Their real self gradually moves away from their ideal self. [...] Owing to the frustration they experience during adolescence, some children give up and lose their goals, and almost act as if their lives are ending. [...] Whatever methods their parents employ, such children do not live a normal life.

Etc1: Seeking monetary profit ${ }^{a}$ without prevalent contextual problems

[While talking about general characteristics of adolescents' PIU] And clients pay for game items. [... ] And third, nowadays, conflicts arise when buying and selling their game accounts [to earn some money for their game items].

${ }^{a}$ Monetary motive was excluded from clustering analysis, because it was one of the minor categories that might have introduced noise when identifying usage types.

Etc2: Seeking something with personal characteristics

Not only psychological problems but also temperamental or physiological characteristics seem to strongly affect [PIU]. For example, sensation seeking [is one such characteristic].

${ }^{\mathrm{b}}$ Personal characteristics were excluded from clustering analysis, because they do not fit the working definition of usage context, which should reveal the way clients perceive their circumstances.

Note. Italicized and bold phrases denote usage motive and usage context, respectively.

$\mathbf{6 5 0}$ | Journal of Behavioral Addictions 7(3), pp. 644-653 (2018) 
Table 4. Quotes from the focus group interview that explain progression patterns between hierarchical relationships

From (1): Academic stress to (2): Inadequate parenting

Parents who have excessive expectations regarding their children's academic performance begin to experience many conflicts with their children [because of the academic pressure], as their children reach puberty. I think this case shows a general trend. (1)

$[\ldots]$

When their children reach middle school, conflicts start because they play a lot of Internet games [and neglect their schoolwork]. Even if the children are not high-risk [but potential-risk] users, because of the parents' anxiety, conflicts become worse. [...] If the clients have a long history of [such] repeated conflicts, it takes a lot of time for them to be normal again. (2)

From (1): Academic stress to (3): Lack of a lifelong goal

Even if the children once did well in school, as they grow older, they face the harsh reality [where their academic performance is no longer good]. They are overwhelmed by the mighty wall of harsh reality, [...] and they realize that their real selves are far from the ideal selves. (1)

$$
[\ldots]
$$

Owing to the frustration they experience during adolescence, some children give up and lose their goals, and almost act as if their lives are ending. [...] [Later] they don't engage in any other social activity [and just use the internet]. There are quite a lot of such cases [that follow this pattern]. (3)

\section{DISCUSSION}

Our research goal was to identify the usage types of PIU by considering motive and context. In this section, we discuss the two main findings derived from our comparison of the characteristics of the six types. Our first main finding is that the context is essential for illustrating usage types, although motive and context were weighted equally. In the clustering analysis, considering both motive and context presented us with a richer picture of the client's state compared to considering only usage motive. For example, if we just considered the motives "to be recognized as best" and "escape from reality," clients in type H3 might seem internally contradictory. However, by considering their context, we can see that these two motives, in fact, form a coherent story. H3 clients are "lacking a lifelong goal" and exhibit "delinquent behavior," and they have problems in "communicating" with their parents. Furthermore, the environment provided by their parents is not affluent, as they show "economic constraints" and an "unstructured" life in terms of after-school activities. Therefore, although H3 clients long to be recognized as human beings, their environment is not ideal, and they wish to escape from being in their current state.

In addition to the clustering analysis, in the focus group interview, the counselors emphasized usage context, because PIU arises within real-world contextual problems. While discussing PIU users' characteristics, counselors defined PIU as a phenomenon "that conceals problems in reality," and emphasized that PIU cannot be eased without directly resolving these problems. These perceptions were also observed when counselors explained the characteristics of clients with PIU: counselors began this explanation with usage context and illustrated usage motives in terms of the context. In addition, as shown in Table 3, the focus group studies emphasized the same motive and context factors that were identified from the clustering analysis.

Our second main finding is that the hierarchical relationships and progression patterns reveal potential developmental paths among different usage contexts. Namely, types belonging to each of the three hierarchical relationships share similar usage context factors. For example, two types belonging to HR2 share two family context factors: inadequate parenting and instability of the caregiver. For the three hierarchical relationships, the progression patterns showed a potential developmental path among different usage contexts, as shown in Table 4: HR1-HR2 and HR1-HR3. The HR1-HR2 progression pattern shows that HR1 migrates to HR2 when inadequate parenting intensifies and leads to conflict. The HR1-HR3 progression pattern shows that HR1 migrates to HR3 when clients lose their lifelong goals because of academic pressure. The HR2-HR3 progression pattern was not found, because development between the corresponding contexts is unlikely. For example, migration from HR2 to HR3 would occur when clients lose their lifelong goals after receiving adequate parenting.

\section{Limitations and implications}

Our results are meaningful in that by incorporating usage context, we extend existing work on the classification of PIU. Specifically, we derived usage types of PIU based on motive and context. Furthermore, we identified patterns of change between types. However, this study has two main limitations. First, although this study shows how usage context plays a key role in identifying types, the data are solely based on a sampling of Korean counseling cases. Thus, our result may not fully capture the PIU phenomenon within Korea. Furthermore, it has been argued that cultural differences can affect PIU (Kuss \& Griffiths, 2012; Lopez-Fernandez et al., 2017). Thus, further studies are needed to examine whether our results are generalizable beyond Korean culture. Second, although this study revealed some hierarchical relationships and progression patterns between types, the data cannot verify whether the actual progression of PIU follows these patterns or not. Since the counseling lasted for only a short period (2 months) for each case and involved treatment for symptoms, we could not observe the natural progression of PIU. Therefore, further studies are needed to investigate whether our results explain the progression of PIU.

Despite these limitations, this study revealed that usage context is key for identifying usage types. As usage context can explain the way users perceive their circumstances, 
understanding it might provide insights into what makes individuals problematically use the Internet. Therefore, to understand the whole picture of PIU, a holistic approach that includes usage context in addition to motive seems necessary.

Funding sources: This work was supported by the National Research Foundation of Korea (NRF) grant funded by the Korea government (MSIT) (no. 2015M3C7A1065859).

Authors' contribution: All authors participated in the concept and design of the research this manuscript is based on. BK was responsible for the statistical analyses and the first draft. BK and SL were responsible for quantifying the counseling cases, had full access to all data in the study, and take responsibility for the integrity of the data and accuracy of the data analyses. YYD contributed to construct theoretical framework. GG supervised the research. YYD and GG contributed substantially to the writing and editing of the manuscript.

Conflict of interest: The authors report no conflict of interest.

\section{REFERENCES}

Bronfenbrenner, U. (1979). The ecology of human development: Experiments by nature and design. Cambridge, MA: Harvard University Press.

Caplan, S. E. (2002). Problematic Internet use and psychosocial well-being: Development of a theory-based cognitivebehavioral measurement instrument. Computers in Human Behavior, 18(5), 553-575. doi:10.1016/S0747-5632(02) 00004-3

Charlton, J. P., \& Danforth, I. D. (2004). Differentiating computerrelated addictions and high engagement. In K. Morgan, J. Sanchez, C. A. Brebbia, \& A. Voiskounsky (Eds.), Human perspectives in the Internet society: Culture, psychology and gender, WIT transactions on information and communication technologies (Vol. 31). Southampton: WIT Press.

Davis, R. A. (2001). A cognitive-behavioral model of pathological Internet use. Computers in Human Behavior, 17(2), 187-195. doi:10.1016/S0747-5632(00)00041-8

Doh, Y. Y., Lee, S., Kim, B., \& Gweon, G. (2018). Cyclic valuecontext reinforcement model of Internet use behavior. Retrieved from http://cclab.snu.ac.kr/@publication

Gainsbury, S. M., King, D. L., Russell, A. M. T., \& Delfabbro, P. (2016). Who pays to play freemium games? The profiles and motivations of players who make purchases within social casino games. Journal of Behavioral Addictions, 5(2), 221-230. doi:10.1556/2006.5.2016.031

Greenacre, M., \& Blasius, J. (Eds.). (2006). Multiple correspondence analysis and related methods. London, UK: CRC Press.

Griffiths, M. D. (2010). The role of context in online gaming excess and addiction: Some case study evidence. International Journal of Mental Health and Addiction, 8(1), 119-125. doi:10.1007/s11469-009-9229-x
Gunuc, S., \& Dogan, A. (2013). The relationships between Turkish adolescents' Internet addiction, their perceived social support and family activities. Computers in Human Behavior, 29(6), 2197-2207. doi:10.1016/j.chb.2013.04.011

Gwet, K. L. (2008). Computing inter-rater reliability and its variance in the presence of high agreement. British Journal of Mathematical and Statistical Psychology, 61(1), 29-48. doi:10.1348/000711006X126600

Huang, Z., Wang, M., Qian, M., Zhong, J., \& Tao, R. (2007). Chinese Internet Addiction Inventory: Developing a measure of problematic Internet use for Chinese college students. CyberPsychology \& Behavior, 10(6), 805-812. doi:10.1089/ cpb.2007.9950

Husson, F., Josse, J., \& Pages, J. (2010). Principal component methods-hierarchical clustering-partitional clustering: Why would we need to choose for visualizing data. Retrieved from http://factominer.free.fr/more/HCPC_husson_josse.pdf

Jun, S., \& Choi, E. (2015). Academic stress and Internet addiction from general strain theory framework. Computers in Human Behavior, 49, 282-287. doi:10.1016/j.chb.2015.03.001

Karlsen, F. (2013). A world of excesses: Online games and excessive playing. Ashgate, UK: Farnham.

Kim, J., LaRose, R., \& Peng, W. (2009). Loneliness as the cause and the effect of problematic Internet use: The relationship between Internet use and psychological well-being. CyberPsychology \& Behavior, 12(4), 451-455. doi:10.1089/ cpb.2008.0327

Kim, M. G., \& Kim, J. (2010). Cross-validation of reliability, convergent and discriminant validity for the Problematic Online Game Use Scale. Computers in Human Behavior, 26(3), 389-398. doi:10.1016/j.chb.2009.11.010

Kneer, J., \& Glock, S. (2013). Escaping in digital games: The relationship between playing motives and addictive tendencies in males. Computers in Human Behavior, 29(4), 1415-1420. doi:10.1016/j.chb.2013.01.030

Ko, C., Yen, J., Yen, C., Chen, C., Weng, C., \& Chen, C. (2008). The association between Internet addiction and problematic alcohol use in adolescents: The problem behavior model. CyberPsychology \& Behavior, 11(5), 571-576. doi:10.1089/ cpb.2007.0199

Kuss, D. J. (2013). Internet gaming addiction: Current perspectives. Psychology Research and Behavior Management, 6, 125-137. doi:10.2147/PRBM.S39476

Kuss, D. J., \& Griffiths, M. D. (2012). Online gaming addiction in children and adolescents: A review of empirical research. Journal of Behavioral Addictions, 1(1), 3-22. doi:10.1556/ JBA.1.2012.1.1

Laconi, S., Tricard, N., \& Chabrol, H. (2015). Differences between specific and generalized problematic Internet uses according to gender, age, time spent online and psychopathological symptoms. Computers in Human Behavior, 48, 236-244. doi:10.1016/j.chb.2015.02.006

Lai, F. T. T., \& Kwan, J. L. Y. (2017). Socioeconomic influence on adolescent problematic Internet use through school-related psychosocial factors and pattern of Internet use. Computers in Human Behavior, 68, 121-136. doi:10.1016/j.chb.2016.11.021

Lee, K., Lee, H. K., Gyeong, H., Yu, B., Song, Y. M., \& Kim, D. (2013). Reliability and validity of the Korean version of the Internet Addiction Test among college students. Journal of Korean Medical Science, 28(5), 763-768. doi:10.3346/jkms. 2013.28.5.763 
Li, D., Li, X., Wang, Y., Zhao, L., Bao, Z., \& Wen, F. (2013). School connectedness and problematic Internet use in adolescents: A moderated mediation model of deviant peer affiliation and self-control. Journal of Abnormal Child Psychology, 41(8), 1231-1242. doi:10.1007/s10802-0139761-9

Liu, Q. X., Fang, X. Y., Deng, L. Y., \& Zhang, J. T. (2012). Parent-adolescent communication, parental Internet use and Internet-specific norms and pathological Internet use among Chinese adolescents. Computers in Human Behavior, 28(4), 1269-1275. doi:10.1016/j.chb.2012.02.010

Lopez-Fernandez, O., Kuss, D. J., Romo, L., Morvan, Y., Kern, L., Graziani, P., Graziani, P., Rousseau, A., Rumpf, H. J., Bischof, A., Gässler, A. K., Schimmenti, A., Passanisi, A., Männikkö, N., Kääriänen, M., Demetrovics, Z., Király, O., Chóliz, M., Zacarés, J. J., Serra, E., Griffiths, M. D., Pontes, H. M., Lelonek-Kuleta, B., Chwaszcz, J., Zullino, D., Rochat, L., Achab, S., \& Billieux, J. (2017). Self-reported dependence on mobile phones in young adults: A European cross-cultural empirical survey. Journal of Behavioral Addictions, 6(2), 168-177. doi:10.1556/2006.6.2017.020

Marino, C., Vieno, A., Altoe, G., \& Spada, M. M. (2017). Factorial validity of the Problematic Facebook Use Scale for adolescents and young adults. Journal of Behavioral Addictions, 6(1), 5-10. doi:10.1556/2006.6.2017.004

Monacis, L., de Palo, V., Griffiths, M. D., \& Sinatra, M. (2016). Validation of the Internet Gaming Disorder Scale - Short-Form (IGDS9-SF) in an Italian-speaking sample. Journal of Behavioral Addictions, 5(4), 683-690. doi:10.1556/2006.5. 2016.083

National Information Society Agency. (2011). Third standardization of Korean Internet Addiction Proneness Scale. Seoul, Korea: National Information Society Agency.

Park, S. K., Kim, J. Y., \& Cho, C. B. (2008). Prevalence of Internet addiction and correlations with family factors among South Korean adolescents. Adolescence, 43(172), 895. Retrieved from https://search.proquest.com/docview/195950665

Pawlikowski, M., Nader, I. W., Burger, C., Stieger, S., \& Brand, M. (2014). Pathological Internet use - It is a multidimensional and not a unidimensional construct. Addiction Research \& Theory, 22(2), 166-175. doi:10.3109/16066359.2013.793313

Smetaniuk, P. (2014). A preliminary investigation into the prevalence and prediction of problematic cell phone use. Journal of Behavioral Addictions, 3(1), 41-53. doi:10.1556/JBA.3.2014.004

Snodgrass, J. G., Dengah, H. F., Lacy, M. G., \& Fagan, J. (2013). A formal anthropological view of motivation models of problematic MMO play: Achievement, social, and immersion factors in the context of culture. Transcultural Psychiatry, 50(2), 235-262. doi:10.1177/1363461513487666

Song, I., LaRose, R., Eastin, M., \& Lin, C. A. (2004). Internet gratifications and Internet addiction: On the uses and abuses of new media. CyberPsychology \& Behavior, 7(4), 384-394. doi:10.1089/cpb.2004.7.384

Wu, A. M. S., Lai, M. H. C., Yu, S., Lau, J. T. F., \& Lei, M.-W. (2017). Motives for Online Gaming Questionnaire: Its psychometric properties and correlation with Internet gaming disorder symptoms among Chinese people. Journal of Behavioral Addictions, 6(1), 11-20. doi:10.1556/2006.6.2017.007

Wu, T., Lin, C., Årestedt, K., Griffiths, M. D., Broström, A., \& Pakpour, A. H. (2017). Psychometric validation of the Persian nine-item Internet Gaming Disorder Scale - Short Form: Does gender and hours spent online gaming affect the interpretations of item descriptions? Journal of Behavioral Addictions, 6(2), 256-263. doi:10.1556/2006.6.2017.025

Yee, N. (2006). Motivations for play in online games. CyberPsychology \& Behavior, 9(6), 772-775. doi:10.1089/ cpb.2006.9.772

Yen, J. Y., Yen, C. F., Chen, C. C., Chen, S. H., \& Ko, C. H. (2007). Family factors of Internet addiction and substance use experience in Taiwanese adolescents. CyberPsychology \& Behavior, 10(3), 323-329. doi:10.1089/cpb.2006.9948

Young, K., Pistner, M., O’Mara, J., \& Buchanan, J. (1999). Cyber disorders: The mental health concern for the new millennium. CyberPsychology \& Behavior, 2(5), 475-479. doi:10.1089/ cpb.1999.2.475

Young, K. S. (1998). Internet addiction: The emergence of a new clinical disorder. CyberPsychology \& Behavior, 1(3), 237-244. doi:10.1089/cpb.1998.1.237 